\title{
Subclinical Celiac Disease Presented with Postpartum Low Back Pain Case Report
}

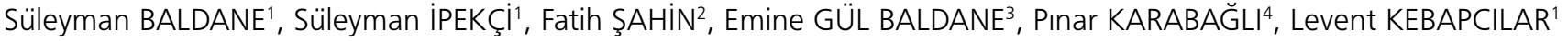 \\ ${ }^{1}$ Department of Endocrinology and Metabolism, Selçuk University Faculty of Medicine, Konya, Turkey \\ ${ }^{2}$ Department of Internal Medicine, Selçuk University Faculty of Medicine, Konya, Turkey \\ ${ }^{3}$ Clinic of Neurology, Konya Meram Training and Research Hospital, Konya, Turkey \\ ${ }^{4}$ Department of Pathology, Selçuk University Faculty of Medicine, Konya, Turkey
}

\begin{abstract}
A 35-year-old woman was admitted with severe low back pain. The patient, who gave birth 5 months ago, was then breastfeeding and presented with postpartum low back pain with increasing severity. Her laboratory results revealed anemia, hypocalcemia, severe vitamin D deficiency, and increase in alkaline phosphatase (ALP) and parathyroid hormone (PTH) levels. Dual energy X-ray absorptiometry (DXA) scan demonstrated lumbar vertebral T and $Z$ scores of -5.1 and -4.4 , respectively. The serologic tests for celiac disease (CD) to search underlying malabsorption were positive. Histological analysis of the duodenal biopsy specimen revealed results consistent with the diagnosis of CD. However, because of an absence of gastrointestinal complaints, the diagnosis of subclinical CD could have been made when malabsorption resulted in osteoporosis associated with severe vitamin $D$ deficiency. The initiation of vitamin $D$ therapy showed a significant increase after 6 months at the lumbar vertebra $T$ and $Z$ scores of -0.7 and -0.2 , respectively.

Keywords: Bone mineral density, celiac disease, osteoporosis, pregnancy
\end{abstract}

\section{Introduction}

Pregnancy-associated osteoporosis, seen in the last trimester or early postpartum period, is a rare condition, and its pathogenesis was not elucidated clearly. Malabsorption conditions, such as celiac disease (CD), and secondary causes of osteoporosis (anorexia nervosa, hyperthyroidism, hyperparathyroidism, Cushing's syndrome, osteogenesis imperfecta, alcoholism, depression etc.) should be investigated in patients with pregnancy-associated osteoporosis (1).

Celiac disease is an autoimmune enteropathy commonly encountered in genetically predisposed individuals and precipitated by the ingestion of gluten-containing foods such as wheat, barley, and rye, which results in malabsorption associated by immune mechanisms (2). Subclinical CD was added to the celiac terminology following the widespread use of serological tests. In this form of the disease that is also called silent $C D$, there are serological findings and mucosal injury similar to the classical form but no gastrointestinal symptoms (3). Patients with subclinical CD may present with non-gastrointestinal findings, such as iron deficiency anemia, teeth enamel coating abnormalities, high level of liver enzymes, and osteoporosis $(3,4)$. The development of osteoporosis may be accelerated in pregnant women with $C D$ due to the increased demand for calcium and increased bone resorption both observed during pregnancy. In this article, 
we present a case of subclinical CD presented with postpartum low back pain in whom we discovered severe lumbar vertebral osteoporosis.

\section{Case Report}

A 35-year-old woman admitted to our neurology clinic with severe low back pain. Then, she was referred to our endocrinology unit because diffuse vertebral signal loss of the lumbar vertebrae was detected in the magnetic resonance imaging (MRI) scan. The patient had given birth five months ago and was breastfeeding. She complained of progressive postpartum low back pain. Her pain increased with movement and decreased with rest. There was no history of trauma, fever, night sweating, abdominal pain, diarrhea, nausea, vomiting, or skin rashes. The patient was receiving no medications apart from the oral iron medication previously prescribed for iron deficiency anemia.

On physical examination, her height was $155 \mathrm{~cm}$, weight was $45 \mathrm{~kg}$, and body mass index was $18.7 \mathrm{~kg} / \mathrm{m}^{2}$; musculoskeletal examination revealed no tenderness in the back or hip joints with a full range of motion in both hip joints and neurological examinations was normal.

The laboratory analyses results were as follows: hemoglobin $10.2 \mathrm{~g} / \mathrm{dL}$ (normal limits: $12.0-16.0 \mathrm{~g} / \mathrm{dL}$ ), serum calcium 7.2 $\mathrm{mg} / \mathrm{dL}$ (normal limits: $8.4-10.2 \mathrm{mg} / \mathrm{dL}$ ), phosphorus $2.0 \mathrm{mg} / \mathrm{dL}$ (normal limits: $2.3-4.7 \mathrm{mg} / \mathrm{dL}$ ), alkaline phosphatase (ALP) 605 $\mathrm{U} / \mathrm{L}$ (normal: $40-150 \mathrm{U} / \mathrm{L}$ ), gamma-glutamyl transferase $10 \mathrm{U} / \mathrm{L}$ (normal limits: 9-36 U/L), $25(\mathrm{OH}$ ) vitamin $\mathrm{D}<5 \mathrm{ng} / \mathrm{mL}$ (normal limits: $30-150 \mathrm{ng} / \mathrm{mL}$ ), and parathyroid hormone (PTH) 640 $\mathrm{pg} / \mathrm{mL}$ (normal limits: 12-65 pg/mL). Thyroid, liver, and renal function tests were within normal limits.

The patient's dual energy X-ray absorptiometry (GE-LUNAR Corp, Madison, USA) scan revealed lumbar vertebral $\left(\mathrm{L}_{1}-\mathrm{L}_{4}\right)$ bone mineral density of $0.597 \mathrm{~g} / \mathrm{m}^{2}$ and $T$ and $Z$ score of -5.1 and -4.4 , respectively. Mild fracture (Grade 1 ) was observed in the $\mathrm{L}_{2}-\mathrm{L}_{3}-\mathrm{L}_{4}$ vertebrae in the X-ray; and hip MRI that was performed to eliminate any possibility of avascular necrosis revealed no pathological findings.

Serological tests were performed to investigate $C D$ associated with malabsorption in the patient who had severe osteoporosis, low calcium, hemoglobin, and vitamin $\mathrm{D}$, and high PTH levels. Anti-gliadin IgG (1/40), anti endomisium IgA-lgG (respectively $1 / 40$ and $1 / 20$ ), and tissue transglutaminase $\lg A-\lg G$ (respectively high positive and low positive) tests were found to be positive. Esophagogastroduodenoscopy demonstrated mucosal hyperemia in the corpus and antral regions of the stomach. A lower duodenal biopsy revealed moderate villous blunting and cript hyperplasia. A significant increase in lamina propria infiltration by lymphoplasmacytic and neutrophilic infiltration were observed. The number of intraepithelial lymphocytes per 100 epithelial cell was 36 (Figure 1, 2).

The diagnosis of subclinical CD was made based on these findings, and the patient was placed on a gluten-free diet and calcium carbonate (1000 $\mathrm{mg}$, three times per day) and vitamin $\mathrm{D}_{3}$ (once $100.000 \mathrm{IU}$ intramuscular and $800 \mathrm{IU}$ one time per day oral drop) treatment. In addition, the patient was recommended to stop breastfeeding, and she gave up breastfeeding before medical treatment.

At the third month follow-up visit, the patient totally recovered from her low back pain and follow-up blood tests revealed hemoglobin level of $12.5 \mathrm{~g} / \mathrm{dL}$, serum calcium of $8.8 \mathrm{mg} / \mathrm{dL}$, phosphorus of $3.9 \mathrm{mg} / \mathrm{dL}$, ALP of $174 \mathrm{U} / \mathrm{L}$, vitamin D of $37.6 \mathrm{ng} /$ $\mathrm{mL}$, and PTH of $52 \mathrm{pg} / \mathrm{mL}$. Bone mineral density measurement 6 months after initiation of therapy using the same machine and read by the same radiologist revealed a significant increase at the lumbar vertebra (L1-L4) $1.072 \mathrm{~g} / \mathrm{m}^{2}$ and $T$ and $Z$ score -0.7 and -0.2 , respectively. Here we report a case of subclinical $C D$ after we obtained the informed consent of the patient.

\section{Discussion}

Celiac disease is an immune mediated, chronic inflammatory disease of the small intestine with gluten sensitivity encountered in genetically susceptible individuals. CD is mostly

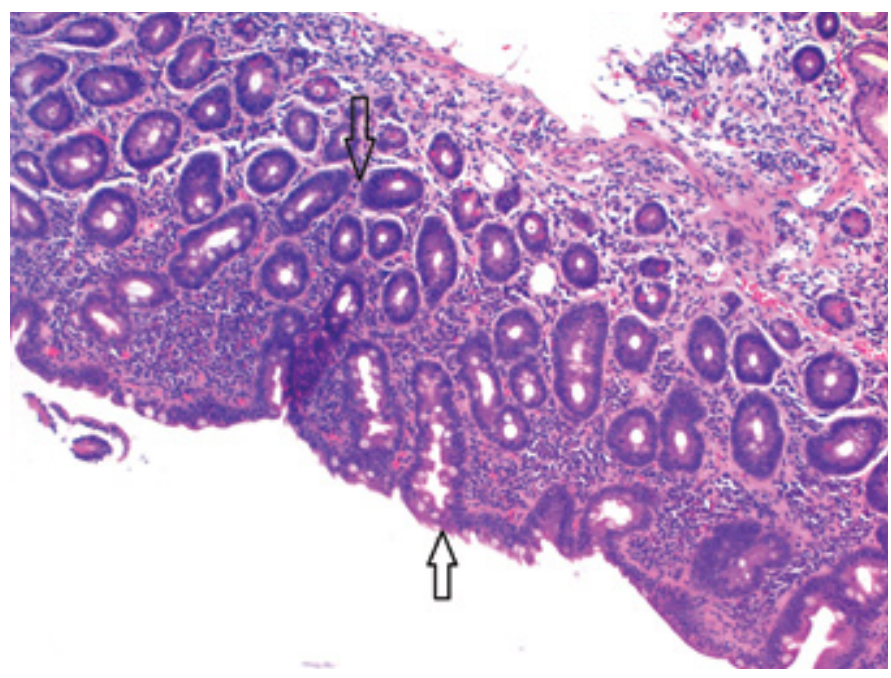

Figure 1. Moderate villous blunting and crypt hyperplasia, HEX100

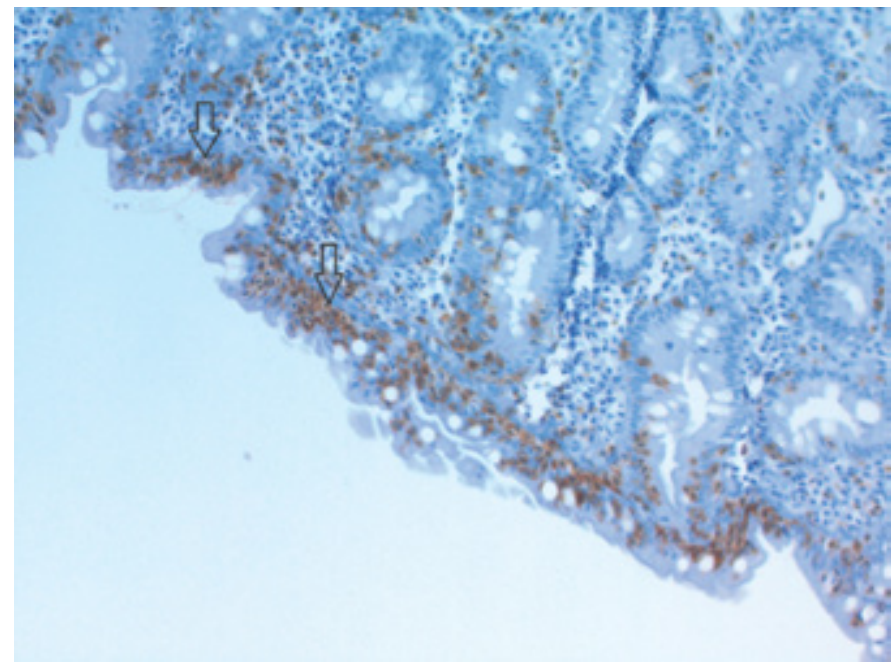

Figure 2. Increased intraepithelial T lymphocytes, CD3X200 
observed in Caucasians. Although it is well known amongst Indian and Pakistani Asians, it is rarely observed among indigenous Africans, Japanese, and Chinese people (5). Many changes regarding serologic data have been reported in the past 30 years because of the availability of more sensitive and relatively cheaper serologic tests. Until 30 years ago, the prevalence of $C D$ in Europe ranged between 1/1000-6500; hence, we considered an uncommon disease. However, in more recent years the prevalence was reported to become $1 / 83-1 / 500$ from the population surveys conducted in various European countries using serological procedures (6-8).

The classification of $C D$ is made according to the presence of gastrointestinal symptoms. The iceberg model of $C D$ was first postulated in multi-centered studies conducted in Italy (9). Patients who present with typical signs of the classical form of $C D$ may be considered as the part of the iceberg above the water, whereas the under-water part comprises 5-10 undiagnosed subclinical or latent CD patients per diagnosed classical CD case (9).

Classical CD presents with signs and symptoms of malabsorption characterized by diarrhea, steatorrhea, abdominal distention, weight loss, and growth failure. In the subclinical form of $C D$, as is the case with the classical disease form, serologic tests are positive and also there is intestinal mucosa injury; however, no gastrointestinal symptom is observed. In this form of the disease, there is limited involvement of the proximal portion of the small intestine. The distal portion is suggested to compensate for the lost functions of the proximal. Therefore, subclinical $C D$ patients may present with non-gastrointestinal findings, such as iron deficiency anemia, teeth enamel coating abnormalities, high level of liver enzymes, and osteoporosis $(3,4)$. Diagnosis of subclinical CD may be incidentally discovered or delayed until the overt symptom of the gastrointestinal system occurs. Therefore, subclinical CD should be considered in patients with non-intestinal findings, such as osteoporosis.

Osteoporosis is a well-known complication of CD. The prevalence of $C D$ in osteoporotic individuals is approximately $3.4 \%$, whereas in non-osteoporotic individuals the prevalence is approximately $0.2 \%(10)$. The classical, subclinical, and latent forms of $\mathrm{CD}$ all may play a role in the development of osteoporosis. Calcium and vitamin $\mathrm{D}$ deficiencies, which relatively occurs secondary to malabsorption, contribute to the development of osteoporosis associated with CD. However, different mechanisms at the receptor level, such as the RANK/RANK-L/ osteoprotegerin system, are currently known to play a role in the development of osteoporosis. The RANK-L is the final mediator of the cytokine (IL-1, TNF, and IL-6) and hormone (such as PTH) network in bone. This soluble polypeptide protein, belonging to the tumor necrosis factor ligand family, is synthesized by osteoblast located in the cell membrane. The system is bound to its RANK receptor, expressed on cells of the osteoclastic line, and stimulates osteoclast maturation, while inhibiting apoptosis. Osteoprotegerin is another component of this system, expressed by osteoblastic cells, inhibits bindings of RANKL/RANK system, maturation of osteoclast, and resorbtion of bone. RANK-L is expressed by inflammatory and normal neutrophils, unlike osteoprotegerin and RANK, which are expressed only by neutrophils exposed to inflammatory processes. Therefore, neutrophils may contribute to activating RANKL/RANK system in subclinical CD (11). These mechanisms may explain osteoporosis in moderate forms of subclinical $C D$ and in latent $C D$ cases with no intestinal mucosa injury. In contrast, the occurrence of early menopause and frequent amenorrhea in patients with $C D$ may also contribute to the development of osteoporosis $(12,13)$.

Calcium absorption and urinary calcium excretion during pregnancy significantly increase compared with the period before pregnancy due to increased fetal calcium demand for fetal bone development. Bone resorption increases during pregnancy; an increase that has been demonstrated by histological and biochemistry tests (14).

Our case was an undiagnosed case of subclinical $C D$ before pregnancy. She was most probably maintaining her bone metabolism without serious osteoporosis development due to the normal functioning of the non-involved distal segment of the intestine before pregnancy. However, hypocalcemia and severe vitamin $\mathrm{D}$ deficiency developed as a result of increased calcium demand during pregnancy that was not adequately provided because of impaired intestinal absorption. Consequently, there was significant bone resorption associated with secondary hyperparathyroidism with consequent development of severe lumbar osteoporosis that caused low back pain.

\section{Conclusion}

Development of osteoporosis may be accelerated in patients with subclinical $C D$ due to the increased calcium demand in pregnancy; hence, patients become symptomatic. Subclinical CD should definitely be investigated in patients with postpartum osteoporosis. Moreover, the fact that dramatic improvement can be obtained in these patients through treatment with calcium and vitamin $\mathrm{D}$ should be kept in mind.

Informed Consent: Written informed consent was obtained patient who participated in this case.

Peer-review: Externally peer-reviewed.

Author Contributions: Concept - S.B., S.I.; Design - S.B., S.I.; Supervision - L.K.; Resource - E.G.B.; Materials - F.Ş., E.G.B.; Data Collection and/or Processing - S.B., S.I.; Analysis and/or Interpretation - S.B., P.K.; Literature Review - S.B., S.I.; Writer - S.B., S.I.; Critical Review - L.K., S.I.

Conflict of Interest: No conflict of interest was declared by the authors.

Financial Disclosure: The authors declared that this study has received no financial support.

\section{References}

1. Di Gregorio S, Danilowicz K, Rubin Z, Mautalen C. Osteoporosis with vertebral fractures associated with pregnancy and lactation. Nutrition 2000;16:1052-5. [CrossRef]

2. Green PH, Cellier C. Celiac disease. N Engl J Med 2007;357:173143. [CrossRef]

3. Ludvigsson JF, Leffler DA, Bai JC, Biagi F, Fasano A, Green PH, et al. The Oslo definitions for coeliac disease and related terms. Gut 2013;62:43-52. [CrossRef] 
4. Tursi A, Giorgetti G, Brandimarte G, Rubino E, Lombardi D, Gasbarrini G. Prevalence and clinical presentation of subclinical/silent celiac disease in adults: an analysis on a 12-year observation. Hepatogastroenterology 2001;48:462-4.

5. Farrel RJ, Kelly CP. Celiac Sprue. N Engl J Med 2002;346:180-8. [CrossRef]

6. Swinson CM, Levi AJ. Is coeliac disease underdiagnosed? $\mathrm{Br}$ Med J 1980;281:1258-60. [CrossRef]

7. Remes-Troche JM, Ramírez-Iglesias MT, Rubio-Tapia A, Alonso-Ramos A, Velazquez A, Uscanga LF. Coeliac disease could be a frequent disease in Mexico: prevalence of tissue transglutaminase antibody in healthy blood donors. J Clin Gastroenterol 2006;40:697-700. [CrossRef]

8. Catassi C, Fabiani E, Rätsch IM, Coppa GV, Giorgi PL, Pierdomenico $\mathrm{R}$, et al. The coeliac iceberg in Italy. A multicentre antigliadin antibodies screening for coeliac disease in school-age subjects. Acta Paediatr Suppl 1996;412:29-35. [CrossRef]
9. Farell RJ, Kelly CP. Diagnosis of celiac sprue. Am J Gastroenterol 2001;96:3237-46. [CrossRef]

10. Stenson WF, Newberry R, Lorenz R, Baldus C, Civitelli R. Increased prevalence of celiac disease and need for routine screening among patients with osteoporosis. Arch Intern Med 2005;165:393-9. [CrossRef]

11. Bianchi ML, Bardella MT. Bone in celiac disease. Osteoporos Int 2008;19:1705-16. [CrossRef]

12. Molteni N, Bardella MT, Bianchi PA. Obstetric and gynecological problems in women with untreated celiac sprue. J Clin Gastroenterol 1990;12:37-9. [CrossRef]

13. Gallagher JC. Effect of early menopause on bone mineral density and fractures. Menopause 2007;14:567-71. [CrossRef]

14. Kalkwarf HJ, Specker BL. Bone mineral changes during pregnancy and lactation. Endocrine 2002;17:49-53. [CrossRef] 